\title{
Differential white cell count and incident type 2 diabetes: the Insulin Resistance Atherosclerosis Study
}

\author{
Carlos Lorenzo • Anthony J. Hanley $\cdot$ Steven M. Haffner
}

Received: 21 August 2013 / Accepted: 17 September 2013 / Published online: 19 October 2013

(C) Springer-Verlag Berlin Heidelberg 2013

\begin{abstract}
Aims/hypothesis White cell count has been shown to predict incident type 2 diabetes, but differential white cell count has received scant attention. We examined the risk of developing diabetes associated with differential white cell count and neutrophil:lymphocyte ratio and the effect of insulin sensitivity and subclinical inflammation on white cell associations.

Methods Incident diabetes was ascertained in 866 participants aged 40-69 years in the Insulin Resistance Atherosclerosis Study after a 5 year follow-up period. The insulin sensitivity index $\left(\mathrm{S}_{\mathrm{I}}\right)$ was measured by the frequently sampled IVGTT. Results C-reactive protein was directly and independently associated with neutrophil $(p<0.001)$ and monocyte counts $(p<0.01)$ and neutrophil:lymphocyte ratio $(p<0.001)$, whereas $\mathrm{S}_{\mathrm{I}}$ was inversely and independently related to lymphocyte count $(p<0.05)$. There were $138(15.9 \%)$ incident cases of diabetes. Demographically adjusted ORs for incident diabetes,
\end{abstract}

Steven M. Haffner has retired from the University of Texas Health Science Center.

Electronic supplementary material The online version of this article (doi:10.1007/s00125-013-3080-0) contains peer-reviewed but unedited supplementary material, which is available to authorised users.

C. Lorenzo $(\bowtie) \cdot$ S. M. Haffner

Department of Medicine, University of Texas Health Science Center,

7703 Floyd Curl Drive, San Antonio, TX 78229, USA

e-mail: lorenzo@uthscsa.edu

A. J. Hanley

Department of Nutritional Sciences and Medicine,

University of Toronto, Toronto, ON, Canada

\section{A. J. Hanley}

Dalla Lana School of Public Health, University of Toronto,

Toronto, ON, Canada

A. J. Hanley

Leadership Sinai Centre for Diabetes, Mt. Sinai Hospital,

Toronto, ON, Canada comparing the top and bottom tertiles of white cell (1.80 [95\% CI 1.10, 2.92]), neutrophil $(1.67[1.04,2.71])$ and lymphocyte counts $(2.30[1.41,3.76])$, were statistically significant. No association was demonstrated for monocyte count $(1.18[0.73$, 1.90]) or neutrophil:lymphocyte ratio $(0.89[0.55,1.45])$. White cell and neutrophil associations were no longer significant after further adjusting for family history of diabetes, fasting glucose and smoking, but the OR comparing the top and bottom tertiles of lymphocyte count remained significant $(1.96[1.13,3.37])$. This last relationship was better explained by $\mathrm{S}_{\mathrm{I}}$ rather than C-reactive protein.

Conclusions/interpretation A lymphocyte association with incident diabetes, which was the strongest association among the major white cell types, was partially explained by insulin sensitivity rather than subclinical inflammation.

Keywords Clinical science $\cdot$ Epidemiology $\cdot$ Human $\cdot$ Insulin sensitivity and resistance $\cdot$ Pathogenic mechanisms .

Prediction and prevention of type 2 diabetes

\begin{tabular}{ll}
\multicolumn{2}{l}{ Abbreviations } \\
AIR & Acute insulin response \\
CRP & C-reactive protein \\
FSIVGTT & Frequently sampled IVGTT \\
hsCRP & High-sensitivity C-reactive protein \\
IRAS & Insulin Resistance Atherosclerosis Study \\
$\mathrm{S}_{\mathrm{I}}$ & Insulin sensitivity index \\
Th17 & IL-17-producing T helper \\
Treg & Foxp3+ regulatory T cell
\end{tabular}

Introduction

Low-grade inflammation is a key component in the pathophysiology of type 2 diabetes [1], particularly in the development of obesity-related insulin resistance [2]. Obesity increases the number of macrophages in adipose tissue and 
upregulates the production of inflammatory factors [3]. In patients with type 2 diabetes, treatment with an IL-1 receptor antagonist and salsalate (a non-acetylated form of salicylate) has been shown to improve glycaemic control and/or beta cell secretory function $[4,5]$. Increased diabetic risk [6] and insulin resistance [7] have been described in patients with chronic inflammatory diseases such as rheumatoid arthritis and psoriasis. Treatment of these conditions with anti-TNF- $\alpha$ blockers ameliorates disease activity, inflammatory mediators and insulin resistance $[8,9]$.

White cell count, a marker of subclinical inflammation, is directly associated with insulin resistance [10-13] and inversely with insulin secretion [11]. White cell count has been shown to predict both worsening insulin sensitivity [10] and incident type 2 diabetes [10, 14-19], although there is controversy on its usefulness in risk prediction [19-21]. Data on the ability to predict type 2 diabetes by major white cell types are scant $[10,15,16]$. A significant association has been reported for both neutrophil and lymphocyte counts, but not for monocyte count $[15,16]$. However, the incidence of diabetes was predicted by white cell count, but not by any major white cell type, in a relatively small study among Pima Indians [10]. Distinct metabolic traits may account (at least partially) for the relationship between white cell subfractions and diabetic risk. Neutrophil count has been shown to correlate with highsensitivity C-reactive protein (hsCRP) concentration better than any other major white cell type in non-diabetic individuals [22]. Lymphocytes are expanded in obese adipose tissue [3] and regulate macrophage production of inflammatory mediators [1]. Raised levels of neutrophils, lymphocytes and the neutrophil:lymphocyte ratio have been linked to the metabolic syndrome [23, 24]. However, whether major white cell types are associated with the future development of type 2 diabetes beyond the effect of insulin sensitivity and subclinical inflammation is not known [16].

The aims of this study were twofold: (1) to examine the risk of developing diabetes associated with total and differential white cell counts and neutrophil:lymphocyte ratio; and (2) to assess the effects of glucose tolerance, insulin sensitivity, insulin secretion and low-grade inflammation on white cell associations. We analysed these issues in 866 participants who were non-diabetic at baseline [25]. Incident diabetes was ascertained after a 5.2 year follow-up using the 2003 ADA diagnostic criteria. The insulin sensitivity index $\left(\mathrm{S}_{\mathrm{I}}\right)$ and acute insulin response (AIR) were directly measured using the frequently sampled IVGTT (FSIVGTT).

\section{Methods}

Study sample The Insulin Resistance Atherosclerosis Study (IRAS) is a multicentre observational epidemiologic study of the relationships between insulin resistance, cardiovascular disease and the known risk factors for insulin resistance in different ethnic groups and varying states of glucose tolerance. The design and methods of this study have previously been described in detail [25]. Briefly, the study was conducted at four clinical centres. At centres in Oakland and Los Angeles, California, non-Hispanic white and African-American participants were recruited from Kaiser Permanente, a non-profit health maintenance organisation. Centres in San Antonio, Texas, and San Luis Valley, Colorado, recruited nonHispanic white and Hispanic participants from two ongoing population-based studies (the San Antonio Heart Study and the San Luis Valley Diabetes Study). The IRAS protocol was approved by local institutional review committees and all participants provided written informed consent.

A total of 1,625 individuals participated in the baseline IRAS examination (56\% women; age range 40-69 years), which occurred between October 1992 and April 1994. After an average of 5.2 years (range 4.5-6.6 years), follow-up examinations were conducted using the baseline protocol. Participants who returned for the follow-up visit (response rate $81 \%$ ) were eligible for analysis if they were nondiabetic at the baseline examination $(n=1,065)$. We excluded participants with no data on incident diabetes (death, $n=22$; no OGTT data at the follow-up visit, $n=153$ ) or white cell count $(n=24)$. Therefore, the present report includes information on 866 participants (222 African-American, 300 Hispanic and 344 non-Hispanic white). These participants were similar to those who were excluded in terms of demographics, baseline metabolic variables and lymphocyte count (all comparisons, $p>0.05$ ), but were different with regard to smoking status and total white cell, neutrophil and monocyte counts (Electronic Supplementary Material [ESM] Table 1). Smoking explained the differences in total white cell and neutrophil counts, but not in monocyte count (ESM Table 2).

Clinical measurements and procedures The IRAS protocol required two visits, 1 week apart, of approximately $4 \mathrm{~h}$ each. Protocols were identical at the baseline and 5 year follow-up examinations. Individuals were asked prior to each visit to fast for $12 \mathrm{~h}$, abstain from heavy exercise and alcohol for $24 \mathrm{~h}$, and refrain from smoking on the morning of the examination. Data on age, sex, race/ethnicity, family history of diabetes, cigarette smoking and medications were gathered by trained personnel. Duplicate measures of anthropometry were made following a standardised protocol, and averages were used in the analyses.

During the first baseline and follow-up visits, a $75 \mathrm{~g}$ OGTT was administered to assess glucose tolerance status. During the second baseline visit, insulin sensitivity and insulin secretion were determined using the FSIVGTT [25]. Some modifications were made to the original FSIVGTT. An injection of regular insulin rather than tolbutamide was used to ensure adequate plasma insulin levels for the accurate computation of insulin sensitivity across a broad range of glucose tolerance. 
Glucose in the form of $50 \%$ solution $(0.3 \mathrm{~g} / \mathrm{kg})$ and regular human insulin $(0.03 \mathrm{U} / \mathrm{kg})$ were injected through an i.v. line at 0 and $20 \mathrm{~min}$, respectively. Blood was collected at $-5,2,4,8$, $19,22,30,40,50,70,100$ and 180 min for measurement of plasma glucose and insulin. $\mathrm{S}_{\mathrm{I}}$ was calculated using mathematical modelling methods (MINMOD program version 3.0 [1994] developed at the laboratory R. Bergman, Department of Physiology and Biophysics, University of Southern California Medical School, Los Angeles, CA, USA). There was a strong degree of agreement between $\mathrm{S}_{\mathrm{I}}$ estimated by minimal model analysis of the insulin-modified FSIVGTT and insulin sensitivity obtained with the euglycaemic-hyperinsulinaemic clamp [26]. AIR was calculated as the mean of 2 and $4 \mathrm{~min}$ insulin concentrations after glucose administration.

The same laboratory carried out analytical procedures for all samples except for complete blood cell counts. Complete blood cell counts were performed with standard techniques (analytical CV $<9 \%$ for total white cell count and differential) [27] at each centre in accredited laboratories [25]. There were no significant differences between instruments (Coulter T540, Beckman-Coulter, Fullerton, CA, USA; Minos STX, Roche Diagnostics, Basel, Switzerland; and H-1 Analyzer, Technicon, Tarrytown, NY, USA) except for lymphocyte count (lower lymphocyte counts using the H-1 Analyzer). Plasma glucose and insulin levels were measured at the central IRAS laboratory at the University of Southern California (Los Angeles, CA, USA). Glucose concentration was determined by the glucose oxidase method (Yellow Springs Equipment Co., Yellow Springs, OH, USA) and insulin concentration by the dextran-charcoal radioimmunoassay (CV 19\%). This assay had a high cross-reactivity with proinsulin [25]. Intact proinsulin concentration was measured at the laboratory of the Department of Clinical Biochemistry at Addenbrooke's Hospital (Cambridge, UK) (CV 14\%) [28]. To determine hsCRP, we used an in-house ultrasensitive competitive immunoassay (antibodies and antigens from Calbiochem, La Jolla, CA, USA) (CV 8.9\%) [12].

Obesity was defined as BMI $\geq 30 \mathrm{~kg} / \mathrm{m}^{2}$. We used the 2003 ADA criteria to define diabetes (fasting glucose $\geq 7.0 \mathrm{mmol} / \mathrm{l}$ and/or $2 \mathrm{~h}$ glucose $\geq 11.1 \mathrm{mmol} / \mathrm{l}$ ) and impaired glucose tolerance ( $2 \mathrm{~h}$ glucose $\geq 7.8$ and $<11.1 \mathrm{mmol} / \mathrm{l})$ [29]. Individuals who reported current treatment with glucose-lowering medications were considered to have diabetes. HOMA-IR was calculated according to Matthew's formula [30]. We grouped participants as never smokers ( $<100$ cigarettes during the lifetime), former smokers ( $>100$ cigarettes during the lifetime but not actively smoking) and current smokers (actively smoking). The proinsulin:insulin ratio was used as a measure of disordered processing of insulin. The neutrophil:lymphocyte ratio was defined as the $\log _{\mathrm{e}}$ neutrophil count $/ \log _{\mathrm{e}}$ lymphocyte count.

Statistical analyses Analyses were carried out using the SAS (version 9.2, SAS Institute, Cary, NC, USA) and R Project statistical software packages (version 2.9.2, The R Foundation for Statistical Computing, Vienna, Austria). We assessed baseline differences in anthropometric variables, selected known risk factors for diabetes and white cell count (including major types of white cells) by diabetic status at follow-up using oneway ANCOVA (for continuous variables) and logistic regression analysis (for dichotomous variables). The strength of the relationship between metabolic risk factors and white cell count (total and individual cell types) and neutrophil:lymphocyte ratio was quantified using Pearson's correlation coefficients and linear regression. The effect of confounders on the relationship between cell counts and incident diabetes was assessed by multiple logistic regression analysis. In separate models, appropriate interaction terms were introduced to assess the impact of sex, race/ethnicity, clinic, family history of diabetes, obesity, smoking and glucose tolerance on the relationship between cell count and incident diabetes. We fitted a different logistic regression model to the data to model incident diabetes with a restricted cubic polynomial spline for total white cell count to estimate the varying effects of total white cell count (or subfractions) over its full range [31]. We used $\log _{\mathrm{e}}$-transformed values of total and differential white cell counts, AIR, proinsulin:insulin ratio, HOMA-IR and hsCRP in all analyses to minimise the influence of extreme observations. We also used the $\log _{e}$ transformation of $\left(\mathrm{S}_{\mathrm{I}}+1\right)$ and (number of cigarettes +1$)$, given that some participants had $\mathrm{S}_{\mathrm{I}}=0$ or did not smoke. We considered $p<0.05$ to be statistically significant.

\section{Results}

The range for white cell count among the 866 non-diabetic participants was $2.1-15.4 \times 10^{9}$ cells/l. The numbers of individuals who were non-smokers, former smokers and active smokers were 405,336 and 125 , respectively. Progression to diabetes was associated with older age and current smoking, but not with sex or race/ethnicity (Table 1). Among smokers, the number of cigarettes smoked per day was not related to incident diabetes. After adjusting for age, sex, race/ethnicity and clinic, progression to diabetes was associated with lower $\mathrm{S}_{\mathrm{I}}$ and AIR, as well as higher adiposity, HOMA-IR, proinsulin:insulin ratio and levels of plasma glucose, fasting insulin and hsCRP. In addition, baseline white cell and lymphocyte counts were higher in individuals who developed diabetes compared with those who had no diabetes at followup. However, no significant differences were demonstrated for neutrophil and monocyte counts, neutrophil:lymphocyte ratio, and white blood cell differential measured as percentage of each type of white blood cell.

After controlling for age, sex, race/ethnicity, clinic and smoking, neutrophils were the white cell type with the strongest correlation to total white cell count (Table 2). Direct correlations were demonstrated between subfractions, but 
Table 1 Baseline characteristics by diabetes status at follow-up
Data are $n$, mean \pm SEM or per cent $(95 \% \mathrm{CI})$

Results are adjusted for age, sex, race/ethnicity and clinic

Neutrophil:lymphocyte ratio $=$ $\log _{e}$-transformed neutrophil count $/ \log _{e}$-transformed lymphocyte count

${ }^{a}$ Non-adjusted values

${ }^{\mathrm{b}} \log _{e}$-transformed values then back-transformed for presentation

${ }^{\mathrm{c}}$ To convert hsCRP to $\mathrm{nM} / \mathrm{l}$, multiply by 9.524

\begin{tabular}{|c|c|c|c|}
\hline Characteristic & No diabetes & Diabetes & $p$ value \\
\hline$n$ & 728 & 138 & - \\
\hline Age (years) ${ }^{a}$ & $54.3 \pm 0.3$ & $56.2 \pm 0.7$ & 0.007 \\
\hline Female $^{\mathrm{a}}$ & $56.5(52.8,60.0)$ & $60.1(51.8,68.0)$ & 0.422 \\
\hline \multicolumn{4}{|l|}{ Ethnicity $^{\mathrm{a}}$} \\
\hline African-American & $25.7(22.6,29.0)$ & $25.4(18.8,33.3)$ & 0.936 \\
\hline Hispanic & $34.1(30.7,37.6)$ & $37.7(30.0,46.0)$ & 0.413 \\
\hline Non-Hispanic white & $40.2(36.7,43.9)$ & $37.0(29.3,45.3)$ & 0.469 \\
\hline \multicolumn{4}{|l|}{ Cigarette smoking $^{\mathrm{a}}$} \\
\hline Never smokers & $48.4(44.7,50.2)$ & $38.4(30.7,46.8)$ & 0.032 \\
\hline Former smokers & $39.3(35.8,42.9)$ & $36.2(28.7,44.6)$ & 0.500 \\
\hline Current smokers & $12.4(10.2,15.0)$ & $25.4(18.8,33.3)$ & $<0.001$ \\
\hline Cigarettes per day among current smokers ${ }^{\mathrm{a}}$ & $15.2 \pm 1.5$ & $14.7 \pm 2.4$ & 0.860 \\
\hline BMI $\left(\mathrm{kg} / \mathrm{m}^{2}\right)$ & $27.9 \pm 0.2$ & $31.0 \pm 0.5$ & $<0.001$ \\
\hline Waist circumference $(\mathrm{cm})$ & $89.1 \pm 0.4$ & $95.6 \pm 1.0$ & $<0.001$ \\
\hline Fasting glucose (mmol/l) & $5.37 \pm 0.02$ & $5.87 \pm 0.04$ & $<0.001$ \\
\hline $2 \mathrm{~h}$ glucose $(\mathrm{mmol} / \mathrm{l})$ & $6.59 \pm 0.07$ & $8.38 \pm 0.14$ & $<0.001$ \\
\hline Fasting insulin $(\mathrm{pmol} / \mathrm{l})^{\mathrm{b}}$ & $71.4 \pm 1.2$ & $105.6 \pm 5.4$ & $<0.001$ \\
\hline HOMA-IR ${ }^{b}$ & $2.83 \pm 0.06$ & $4.57 \pm 0.28$ & $<0.001$ \\
\hline $\mathrm{S}_{\mathrm{I}}\left(\times 10^{-5} \mathrm{~min}^{-1} \mathrm{pmol}^{-1} 1^{-1}\right)^{\mathrm{b}}$ & $3.21 \pm 0.10$ & $1.75 \pm 0.18$ & $<0.001$ \\
\hline $\operatorname{AIR}(\mathrm{pmol} / \mathrm{l})^{\mathrm{b}}$ & $318.0 \pm 9.6$ & $235.8 \pm 16.8$ & $<0.001$ \\
\hline AIR adjusted for $\mathrm{S}_{\mathrm{I}}(\mathrm{pmol} / \mathrm{l})^{\mathrm{b}}$ & $327.6 \pm 10.2$ & $198.6 \pm 12.0$ & $<0.001$ \\
\hline Proinsulin:insulin ratio $(\times 100)^{\mathrm{b}}$ & $6.08 \pm 0.12$ & $6.93 \pm 0.36$ & 0.029 \\
\hline $\mathrm{hsCRP}(\mathrm{mg} / \mathrm{l})^{\mathrm{bc}}$ & $1.67 \pm 0.07$ & $2.61 \pm 0.25$ & $<0.001$ \\
\hline White cell count $\left(\times 10^{9} / 1\right)^{\mathrm{b}}$ & $5.39 \pm 0.06$ & $5.78 \pm 0.14$ & 0.007 \\
\hline Neutrophils (\%) & $59.8 \pm 0.3$ & $59.7 \pm 0.3$ & 0.993 \\
\hline Lymphocytes (\%) & $32.0 \pm 0.3$ & $32.3 \pm 0.7$ & 0.670 \\
\hline Monocytes (\%) & $6.06 \pm 0.10$ & $5.76 \pm 0.22$ & 0.197 \\
\hline Neutrophil count $\left(\times 10^{9} / 1\right)^{\mathrm{b}}$ & $3.11 \pm 0.05$ & $3.33 \pm 0.11$ & 0.064 \\
\hline Lymphocyte count $\left(\times 10^{9} / 1\right)^{\mathrm{b}}$ & $1.65 \pm 0.02$ & $1.81 \pm 0.05$ & $<0.001$ \\
\hline Monocyte count $\left(\times 10^{9} / 1\right)^{\mathrm{b}}$ & $0.30 \pm 0.01$ & $0.31 \pm 0.01$ & 0.318 \\
\hline Neutrophil:lymphocyte ratio ${ }^{\text {b }}$ & $1.087 \pm 0.002$ & $1.082 \pm 0.005$ & 0.349 \\
\hline
\end{tabular}

were relatively weak. Neutrophil:lymphocyte ratio had a strong relationship with neutrophil and lymphocyte counts (direct and inverse, respectively). White cell counts and subfractions tended to have weak correlations with measures of adiposity, insulin resistance/sensitivity and subclinical inflammation. Proinsulin:insulin ratio was not related to any white cell type. The weak relationship between AIR and total white cell and lymphocyte counts was partially explained by $\mathrm{S}_{\mathrm{I}}$. None of the metabolic markers except hsCRP had a significant relationship with neutrophil:lymphocyte ratio. Similar results were obtained without controlling for the effect of any covariate (ESM Table 3).

Additional analyses were carried out to further assess the relationship between established risk factors for diabetes (plasma glucose, fasting insulin, $\mathrm{S}_{\mathrm{I}}$, AIR and hsCRP) and total white cell count and subfractions (Table 3). Fasting insulin rather than $\mathrm{S}_{\mathrm{I}}$ had a consistent and independent relationship with total white cell count and subfractions except for neutrophils. In addition, hsCRP had a consistent and independent relationship with total white cell count and subfractions except for lymphocytes. Two additional independent relationships were demonstrated: BMI with lymphocyte count and $2 \mathrm{~h}$ glucose with total white cell and neutrophil counts. The only independent correlate of neutrophil:lymphocyte ratio was hsCRP.

A total of 138 participants (15.9\%) developed diabetes. The diagnosis was made in $126(91.3 \%)$ of these participants by OGTT criteria. The other 12 participants were already receiving glucose-lowering medications. Figure 1 presents the relationship between incident diabetes and total white cell count and subfractions modelled by a smooth function. Results were adjusted for age, sex, race/ethnicity and clinic. The relationship was linear for all cell types (Wald test for linearity, $p>0.3$ ). It was statistically significant for total white cell count 
Table 2 Pearson partial correlation coefficients between total white cell counts and subfractions and selected metabolic risk factors at baseline

\begin{tabular}{|c|c|c|c|c|c|}
\hline Variable & $\begin{array}{l}\log _{e} \text { white } \\
\text { cell count }\end{array}$ & $\begin{array}{l}\log _{e} \text { neutrophil } \\
\text { count }\end{array}$ & $\begin{array}{l}\log _{e} \text { lymphocyte } \\
\text { count }\end{array}$ & $\begin{array}{l}\log _{e} \text { monocyte } \\
\text { count }\end{array}$ & $\begin{array}{l}\text { Neutrophil: } \\
\text { lymphocyte ratio }\end{array}$ \\
\hline $\log _{e}$ neutrophil count & $0.92^{* * *}$ & - & - & - & - \\
\hline $\log _{e}$ lymphocyte count & $0.54^{* * *}$ & $0.22^{* * *}$ & - & - & - \\
\hline $\log _{e}$ monocyte count & $0.46^{* * *}$ & $0.35^{* * *}$ & $0.21^{* * *}$ & - & - \\
\hline Neutrophil:lymphocyte ratio & $0.39^{* * *}$ & $0.69^{* * *}$ & $-0.55^{* * *}$ & $0.15^{* * *}$ & - \\
\hline Fasting glucose & $0.11^{* *}$ & $0.08^{*}$ & $0.12^{* * *}$ & 0.02 & -0.02 \\
\hline $2 \mathrm{~h}$ glucose & $0.20^{* * *}$ & $0.18^{* * *}$ & $0.15^{* * *}$ & $0.08^{*}$ & 0.04 \\
\hline BMI & $0.19^{* * *}$ & $0.15^{* * *}$ & $0.20^{* * *}$ & $0.07^{*}$ & -0.02 \\
\hline Waist circumference & $0.19^{* * *}$ & $0.14^{* * *}$ & $0.20^{* * *}$ & 0.06 & -0.03 \\
\hline $\log _{e}$ fasting insulin & $0.25^{* * *}$ & $0.17^{* * *}$ & $0.25^{* * *}$ & $0.12^{* * *}$ & -0.04 \\
\hline $\log _{e}$ HOMA-IR & $0.25^{* * *}$ & $0.17^{* * *}$ & $0.25^{* * *}$ & $0.12^{* * *}$ & -0.04 \\
\hline $\log _{e} \mathrm{~S}_{\mathrm{I}}$ & $-0.22^{* * *}$ & $-0.18^{* * *}$ & $-0.22^{* * *}$ & $-0.08^{*}$ & 0.01 \\
\hline $\log _{e} \mathrm{AIR}$ & $0.09^{*}$ & 0.07 & $0.09^{*}$ & 0.07 & 0.00 \\
\hline $\log _{e} \mathrm{AIR}^{\mathrm{a}}$ & 0.02 & 0.00 & 0.01 & 0.05 & 0.00 \\
\hline $\log _{e}$ proinsulin:insulin ratio & -0.05 & -0.02 & -0.06 & -0.03 & 0.03 \\
\hline $\log _{e} \mathrm{hsCRP}$ & $0.28^{* * *}$ & $0.27^{* * *}$ & $0.13^{* * *}$ & $0.13^{* * *}$ & $0.13^{* * *}$ \\
\hline
\end{tabular}

Pearson partial correlation coefficients controlling for age, sex, race/ethnicity, clinic and $\log _{e}$-cigarettes currently smoked per day ${ }^{*} p<0.05 ;{ }^{* *} p<0.01 ;{ }^{* * *} p<0.001$

${ }^{a}$ Pearson partial correlation coefficients also controlling for $\log _{e} \mathrm{~S}_{\mathrm{I}}$

Table 3 Relationship between selected metabolic variables and total and individual white cell type counts (dependent variable) by multiple linear regression analyses

\begin{tabular}{|c|c|c|c|c|c|}
\hline Variable & $\begin{array}{l}\log _{e} \text { white cell } \\
\text { count } \beta(95 \% \mathrm{CI})\end{array}$ & $\begin{array}{l}\log _{e} \text { neutrophil } \\
\text { count } \beta(95 \% \mathrm{CI})\end{array}$ & $\begin{array}{l}\log _{e} \text { lymphocyte } \\
\text { count } \beta(95 \% \mathrm{CI})\end{array}$ & $\begin{array}{l}\log _{e} \text { monocyte } \\
\text { count } \beta(95 \% \mathrm{CI})\end{array}$ & $\begin{array}{l}\text { Neutrophil:lymphocyte } \\
\text { count } \beta(95 \% \mathrm{CI})\end{array}$ \\
\hline \multicolumn{6}{|l|}{ Adjustment model 1} \\
\hline BMI & $0.005 \pm 0.011$ & $0.006 \pm 0.016$ & $0.025 \pm 0.012^{*}$ & $-0.008 \pm 0.019$ & $-0.003 \pm 0.003$ \\
\hline Fasting glucose & $0.003 \pm 0.010$ & $0.003 \pm 0.015$ & $-0.005 \pm 0.011$ & $-0.005 \pm 0.018$ & $0.001 \pm 0.002$ \\
\hline $\log _{e}$ fasting insulin & $0.047 \pm 0.011^{* * *}$ & $0.043 \pm 0.015^{* *}$ & $0.057 \pm 0.012^{* * *}$ & $0.049 \pm 0.018^{* *}$ & $-0.003 \pm 0.002$ \\
\hline $\log _{e} \mathrm{hsCRP}$ & $0.059 \pm 0.010^{* * *}$ & $0.079 \pm 0.015^{* * *}$ & $0.014 \pm 0.011$ & $0.045 \pm 0.018^{*}$ & $0.009 \pm 0.002^{* * *}$ \\
\hline \multicolumn{6}{|l|}{ Adjustment model 2} \\
\hline BMI & $0.007 \pm 0.011$ & $0.000 \pm 0.016$ & $0.027 \pm 0.012^{*}$ & $-0.002 \pm 0.019$ & $-0.004 \pm 0.003$ \\
\hline $2 \mathrm{~h}$ glucose & $0.031 \pm 0.011^{* *}$ & $0.049 \pm 0.016^{* *}$ & $0.014 \pm 0.013$ & $0.039 \pm 0.020^{*}$ & $0.005 \pm 0.003$ \\
\hline $\log _{e} \mathrm{~S}_{\mathrm{I}}$ & $-0.018 \pm 0.013$ & $-0.011 \pm 0.018$ & $-0.036 \pm 0.014^{*}$ & $0.005 \pm 0.022$ & $0.004 \pm 0.003$ \\
\hline $\log _{e} h_{S C R P}$ & $0.061 \pm 0.011^{* * *}$ & $0.087 \pm 0.015^{* * *}$ & $0.011 \pm 0.012$ & $0.048 \pm 0.018^{* *}$ & $0.010 \pm 0.002^{* * *}$ \\
\hline $\log _{e} \mathrm{AIR}$ & $0.013 \pm 0.011$ & $0.018 \pm 0.015$ & $0.011 \pm 0.012$ & $0.034 \pm 0.019$ & $0.001 \pm 0.002$ \\
\hline \multicolumn{6}{|l|}{ Adjustment model 3} \\
\hline BMI & $-0.001 \pm 0.011$ & $-0.006 \pm 0.016$ & $0.017 \pm 0.013$ & $-0.011 \pm 0.020$ & $-0.003 \pm 0.003$ \\
\hline $2 \mathrm{~h}$ glucose & $0.028 \pm 0.011^{* *}$ & $0.043 \pm 0.015^{* *}$ & $0.011 \pm 0.012$ & $0.029 \pm 0.018$ & $0.004 \pm 0.002$ \\
\hline $\log _{e}$ fasting insulin & $0.042 \pm 0.012^{* * *}$ & $0.033 \pm 0.017$ & $0.050 \pm 0.013^{* * *}$ & $0.056 \pm 0.021^{* *}$ & $-0.003 \pm 0.003$ \\
\hline $\log _{e} \mathrm{~S}_{\mathrm{I}}$ & $-0.004 \pm 0.013$ & $-0.003 \pm 0.019$ & $-0.017 \pm 0.015$ & $0.017 \pm 0.023$ & $0.002 \pm 0.003$ \\
\hline $\log _{e}$ hsCRP & $0.058 \pm 0.011^{* * *}$ & $0.085 \pm 0.015^{* * *}$ & $0.007 \pm 0.012$ & $0.045 \pm 0.018^{*}$ & $0.010 \pm 0.002^{* * *}$ \\
\hline
\end{tabular}

Adjustment model 1: age, sex, race/ethnicity, clinic, family history of diabetes, $\log _{e}$-cigarette smoking, cardiovascular disease, emphysema, treatment with medications, BMI, fasting glucose, fasting insulin and $\log _{e}$ hsCRP were all included as independent variables in all five regression models Adjustment model 2: all variables of adjustment model 1, but $2 \mathrm{~h}$ glucose, $\log _{e} \mathrm{~S}_{\mathrm{I}}$ and $\log _{e}$ AIR were substituted for fasting glucose and insulin Adjustment model 3: all variables of adjustment model 1, but $2 \mathrm{~h}$ glucose and $\log _{e} \mathrm{~S}_{\mathrm{I}}$ were substituted for fasting glucose Variable estimates expressed per $1 \mathrm{SD}$ unit increase; ${ }^{*} p<0.05 ;{ }^{* *} p<0.01 ;{ }^{* * *} p<0.001$ 

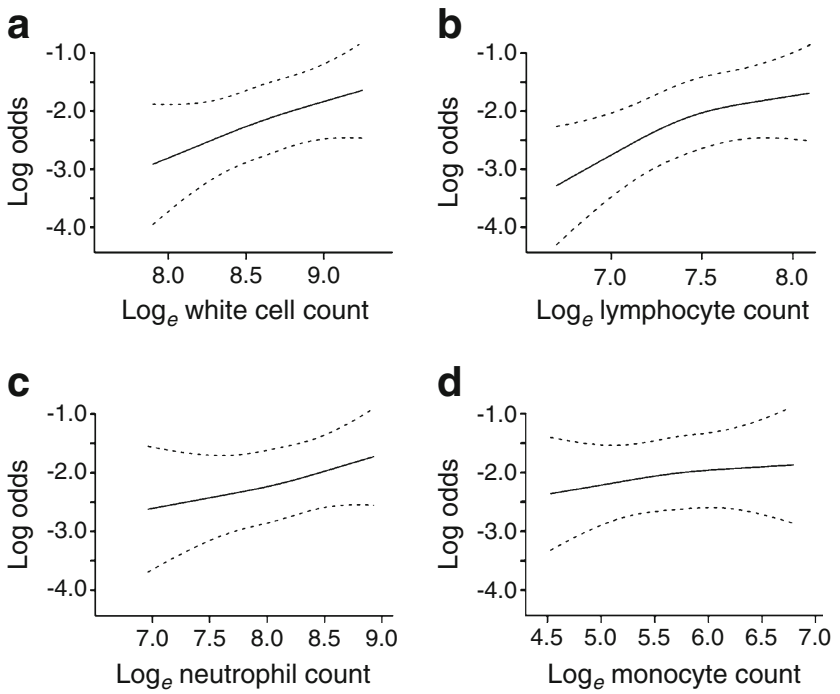

Fig. 1 Relationship between 5 year risk of type 2 diabetes and total white cell count and subfractions modelled by a smooth function. The relationship was linear for all cell types (Wald for linearity, $p>0.3$ ) and statistically significant for (a) white cell count ( $p$ value of the Wald $\chi^{2}=0.028$ ) and (b) lymphocyte count $(p=0.004)$. (c) Neutrophil count $(p=0.161)$ and (d) monocyte count $(p=0.600)$ were not associated with incident diabetes. Results were adjusted for age, sex, race/ethnicity and clinic

$(\mathrm{OR} \times 1 \mathrm{SD}$ unit increase, 1.40 [95\% CI 1.09, 1.80], $p=0.028)$ and lymphocyte count (OR 1.59 [1.20, 2.09], $p=0.004$ ), but not for neutrophil count (OR 1.21 [0.99, 1.60], $p=0.161$ ) or monocyte count (OR $1.12[0.88,1.43], p=0.600)$. None of the white cell types expressed as percentage of total white cell count was associated with incident diabetes (data not shown).

We also examined ORs (95\% CIs) of incident diabetes comparing middle and upper tertiles with the lower tertile of total white cell, absolute neutrophil, lymphocyte and monocyte counts, and neutrophil:lymphocyte ratio by multiple logistic regression analyses (Table 4). Associations for white cell and neutrophil counts were largely explained by the effect of family history of diabetes, fasting glucose and smoking. ORs for lymphocyte count increased in a stepwise fashion. The lymphocyte association was partially explained by insulin sensitivity/resistance $\left(\mathrm{S}_{\mathrm{I}}\right.$, fasting insulin or HOMA-IR) or $2 \mathrm{~h}$ glucose, but not by fasting glucose, smoking, BMI, AIR or hsCRP. Monocyte count and neutrophil:lymphocyte ratio were not associated with incident diabetes. A more comprehensive analysis is presented in ESM Table 4.

In separate models, we examined the effect of sex, race/ ethnicity, clinic, smoking, obesity and glucose tolerance status on the relationship between white cell and lymphocyte counts and incident diabetes (Fig. 2). None of the interaction terms was significant for either total white cell count models or lymphocyte count models. Even though interaction terms smoking $\times$ lymphocyte count and smoking $\times$ white cell count were not statistically significant, neither white cell count nor lymphocyte count was associated with progression to diabetes in current smokers. In addition, none of the interaction terms was statistically significant in models that examined the relationship between neutrophil count and incident diabetes (data not shown).

\section{Discussion}

This study has several novel findings. In non-diabetic individuals, lymphocyte count was associated with insulin sensitivity; neutrophil and monocyte counts with subclinical inflammation, as measured by hsCRP; and total white cell count with both insulin sensitivity and subclinical inflammation. The lymphocyte was the white cell type with the strongest relationship to incident diabetes, some of which is explained by insulin resistance rather than low-grade inflammation.

Many studies have reported a significant relationship between white cell count and type 2 diabetes [10,14-19], but a concern of publication bias has been raised by GkraniaKlotsas et al in a systematic review and meta-analysis [16]. In addition, the confounding effect of established risk factors for type 2 diabetes other than family history of diabetes, adiposity and/or fasting glucose has not been examined [14-19], except for in the study by Vozarova et al [10]. Among 272 Pima Indians with normal glucose tolerance at baseline, the study by Vozarova et al demonstrated an independent relationship between white cell count and incident diabetes after controlling for adiposity, insulin action (measured by the hyperinsulinaemic clamp) and AIR [10]. Despite the evidence relating white cell count to incident diabetes, attempts to include white cell count in risk-prediction models have produced mixed results [19-21]. Our data link white cell count to the development of diabetes, but side with studies reporting a limited ability of white cell count to reclassify individuals according to their risk of diabetes [20,21]. In the IRAS, white cell count was not associated with incident diabetes beyond the effect of family history of diabetes, smoking and fasting glucose.

Few studies have examined the relationship between differential white cell count and incidence of diabetes. Among 12,330 middle-aged participants in the Atherosclerosis Risk in Communities study, neutrophil and lymphocyte counts were associated with incident type 2 diabetes [15]. Similar results were obtained among the 15,550 participants in the EPIC-Norfolk study [16]. Both of these studies adjusted their results for smoking, family history of diabetes, physical activity and adiposity (and fasting glucose in the Atherosclerosis Risk in Communities study), but neither study examined the impact of insulin resistance, glucose tolerance, insulin secretion or subclinical inflammation. In contrast to these reports, Vozarova et al described no significant relationship between neutrophil and lymphocyte counts and incident diabetes in 154 Pima Indians with normal glucose tolerance at baseline [10]. In 
Table 4 Risk of developing diabetes associated with total white cell count and subfractions

\begin{tabular}{|c|c|c|c|c|c|}
\hline Variable & Adjustment model & Lower tertile & Middle tertile & Upper tertile & $p$ for trend \\
\hline \multicolumn{6}{|c|}{ White cell count } \\
\hline Model 1 & Demographics (age, sex, ethnicity and clinic) & 1.00 & $1.70(1.06,2.74)$ & $1.80(1.10,2.92)$ & 0.020 \\
\hline Model 2 & Demographics + family history + fasting glucose & 1.00 & $1.61(0.96,2.70)$ & $1.52(0.89,2.59)$ & 0.141 \\
\hline Model 3 & Demographics + family history + fasting glucose + smoking & 1.00 & $1.44(0.86,2.44)$ & $1.15(0.66,2.02)$ & 0.683 \\
\hline \multicolumn{6}{|c|}{ Neutrophil count } \\
\hline Model 1 & Demographics (age, sex, ethnicity and clinic) & 1.00 & $1.42(0.87,2.30)$ & $1.67(1.04,2.71)$ & 0.037 \\
\hline Model 2 & Demographics + family history + fasting glucose & 1.00 & $1.30(0.76,2.21)$ & $1.51(0.89,2.56)$ & 0.127 \\
\hline Model 3 & Demographics + family history + fasting glucose + smoking & 1.00 & $1.18(0.69,2.02)$ & $1.17(0.67,2.03)$ & 0.607 \\
\hline \multicolumn{6}{|c|}{ Lymphocyte count } \\
\hline Model 1 & Demographics (age, sex, ethnicity and clinic) & 1.00 & $1.73(1.06,2.83)$ & $2.30(1.41,3.76)$ & $<0.001$ \\
\hline Model 2 & Demographics + family history + fasting glucose & 1.00 & $1.71(1.00,2.93)$ & $2.20(1.29,3.76)$ & 0.004 \\
\hline Model 3 & Demographics + family history + fasting glucose + smoking & 1.00 & $1.54(0.89,2.66)$ & $1.96(1.13,3.37)$ & 0.017 \\
\hline Model 4 & Model 3+BMI & 1.00 & $1.59(0.92,2.75)$ & $1.88(1.08,3.25)$ & 0.028 \\
\hline Model 5 & Model $3+2 \mathrm{~h}$ glucose & 1.00 & $1.44(0.81,2.56)$ & $1.64(0.92,2.91)$ & 0.098 \\
\hline Model 6 & Model $3+\log _{\mathrm{e}}$ fasting insulin & 1.00 & $1.54(0.88,2.67)$ & $1.69(0.97,2.96)$ & 0.074 \\
\hline Model 7 & Model $3+\log _{\mathrm{e}}$ HOMA-IR & 1.00 & $1.54(0.88,2.67)$ & $1.69(0.97,2.96)$ & 0.074 \\
\hline Model 8 & Model $3+\log _{\mathrm{e}} \mathrm{S}_{\mathrm{I}}$ & 1.00 & $1.38(0.78,2.45)$ & $1.44(0.80,2.58)$ & 0.242 \\
\hline Model 9 & Model $3+\log _{\mathrm{e}}$ AIR & 1.00 & $1.53(0.88,2.69)$ & $2.02(1.15,3.56)$ & 0.014 \\
\hline Model 10 & Model $3+\log _{\mathrm{e}} \mathrm{S}_{\mathrm{I}}+\log _{\mathrm{e}}$ AIR & 1.00 & $1.31(0.73,2.35)$ & $1.47(0.81,2.67)$ & 0.214 \\
\hline Model 11 & Model $3+\log _{\mathrm{e}}$ hsCRP & 1.00 & $1.62(0.93,2.82)$ & $1.89(1.08,3.30)$ & 0.028 \\
\hline \multicolumn{6}{|c|}{ Monocyte count } \\
\hline Model 1 & Demographics (age, sex, ethnicity and clinic) & 1.00 & $1.26(0.79,2.01)$ & $1.18(0.73,1.90)$ & 0.506 \\
\hline Model 2 & Demographics + family history + fasting glucose & 1.00 & $1.34(0.80,2.23)$ & $1.27(0.76,2.12)$ & 0.382 \\
\hline Model 3 & Demographics + family history + fasting glucose + smoking & 1.00 & $1.32(0.79,2.22)$ & $1.13(0.67,1.92)$ & 0.671 \\
\hline \multicolumn{6}{|c|}{ Neutrophil:lymphocyte ratio } \\
\hline Model 1 & Demographics (age, sex, ethnicity and clinic) & 1.00 & $1.29(0.81,2.03)$ & $0.89(0.55,1.45)$ & 0.618 \\
\hline Model 2 & Demographics + family history + fasting glucose & 1.00 & $1.21(0.74,1.98)$ & $0.85(0.50,1.45)$ & 0.540 \\
\hline Model 3 & Demographics + family history + fasting glucose + smoking & 1.00 & $1.15(0.70,1.89)$ & $0.74(0.43,1.27)$ & 0.257 \\
\hline
\end{tabular}

Values are OR $(95 \% \mathrm{CI})$ expressed per $1 \mathrm{SD}$ unit increase

Range for total and differential white cell count tertiles $\left(\times 10^{9} / 1\right)$ : white cell count: lower $2.1-4.9$, middle 5-6.2, upper 6.3-15.4; neutrophil count: lower 0.41-2.82, middle 2.83-3.80, upper 3.81-11.5; lymphocyte count: lower 0.55-1.47, middle 1.48-1.92, upper 1.93-4.18; monocyte count: lower 0-0.26, middle $0.27-0.36$, upper $0.37-1.13$

Range for neutrophil:lymphocyte ratio tertiles: lower 0.78-1.06, middle 1.07-1.11, upper 1.12-1.42

Family history indicates family history of diabetes; smoking was expressed as $\log _{e}$-cigarettes currently smoked per day

our study, the lymphocyte appears to be the major white cell type that is associated with incident diabetes. The results were consistent across varying categories of sex, race/ethnicity, adiposity and glucose tolerance. This association remained significant after controlling for factors that influence white cell count and/or diabetes risk (e.g. smoking, family history of diabetes, fasting glucose and BMI). It is partially explained by insulin sensitivity rather than subclinical inflammation. On the other hand, the neutrophil association is largely explained by family history of diabetes, fasting glucose and smoking.

White cell count is one of the markers of subclinical inflammation linked to the metabolic syndrome [11-13]. Several studies have described a relationship between white cell count and insulin action measured by the hyperinsulinaemic clamp
$[10,13,32]$. The association with insulin secretion is less well established: raised white cell count has been related to lower insulinogenic index [11], but not to lower AIR [10]. In the latter study, elevated white cell count was also associated with a longitudinal decline in insulin action, but not with worsening AIR [10]. In addition, the relationships of white cell count to adiposity [15, 21, 32] and fasting insulin levels [32-34] are well described. White cell count has been independently related to glucose tolerance and subclinical inflammation (fibrinogen) in multiple regression analyses [34]. Our results suggest that white cell count has an independent relationship with markers of insulin resistance, glucose tolerance and subclinical inflammation. The association with fasting insulin concentration is not fully explained by $\mathrm{S}_{\mathrm{I}}$, a direct measure of 


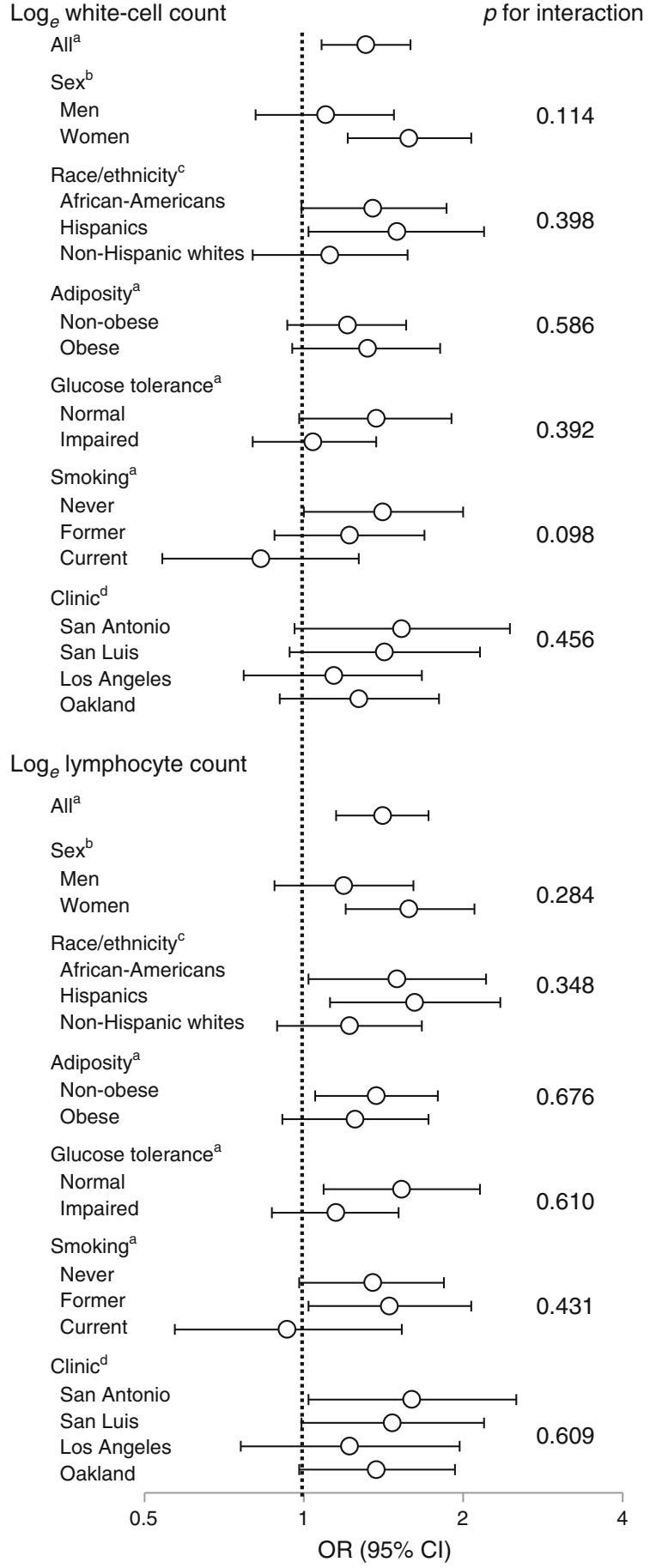

Fig. 2 Heterogeneity analyses for the relationship of white cell and lymphocyte counts to the 5 year incidence of diabetes. Results adjusted for ${ }^{a}$ age, sex, race/ethnicity and clinic; ${ }^{b}$ age, race/ethnicity and clinic; ${ }^{c}$ age, sex and clinic; and ${ }^{d}$ age, sex and race/ethnicity

insulin resistance. In the IRAS, white cell count was not independently associated with adiposity and measures of insulin secretion such as AIR and proinsulin:insulin ratio, a marker of beta cell stress.

Our cross-sectional analysis revealed a distinctive metabolic pattern of relationships for each white cell subfraction: neutrophil and monocyte counts with subclinical inflammation (measured by hsCRP) and glucose tolerance; and lymphocyte count with insulin sensitivity/resistance and adiposity. Although neutrophil count has tended to be associated with subclinical inflammation [22,34], the relationship of neutrophil count to insulin resistance [22], and that of lymphocyte count to subclinical inflammation [22] and insulin resistance and BMI [34], has been less consistent. However, these studies have generally had relatively small sample sizes with which to carry out a comprehensive analysis of the effect of major confounders. In the IRAS, post-load plasma glucose and subclinical inflammation appeared to account for the relationship between neutrophil count and insulin resistance, whereas insulin resistance accounted for the relationship of lymphocyte count to plasma glucose and hsCRP.

A notable number of studies have favoured the use of the neutrophil:lymphocyte ratio as a marker of inflammation, for example, to predict survival in patients with myocardial infarction [35] and progression to steatohepatitis in those with non-alcoholic fatty liver disease [36]. In apparently healthy non-diabetic individuals, a raised neutrophil:lymphocyte ratio has been associated with the metabolic syndrome and elevated hsCRP [24]. In our study, the neutrophil:lymphocyte ratio linked only to subclinical inflammation as measured by hsCRP, and was not associated with progression to diabetes. The absence of a direct relationship between neutrophil:lymphocyte ratio and insulin resistance (and adiposity) may reflect concurrent recruitment of cells of myeloid and lymphoid lineage in the adipose tissue with weight gain [37]. Thus, abnormal neutrophil:lymphocyte ratios should be explained by processes other than adiposity and insulin resistance.

Increased numbers of macrophages, neutrophils, $\mathrm{T}$ cells, $\mathrm{B}$ cells and mast cells infiltrate adipose tissue with weight gain, and appear to play an essential role in insulin resistance [37]. Macrophages become activated and secrete proinflammatory cytokines such as IL-6, TNF- $\alpha$ and IL- $1 \beta$, which can contribute to insulin resistance [38]. The adaptive immune system also has an important role in metabolic regulation and type 2 diabetes [37]. T cell infiltration has been shown to precede the recruitment of macrophages in an experimental model of obesity and to correlate with waist circumference in individuals with type 2 diabetes [39]. The production of TNF- $\alpha$, IFN- $\gamma$ and IL- 6 by activated T cells, both Thelper type 1 cells and cytotoxic $\mathrm{CD} 8+\mathrm{T}$ cells, contributes to metabolic dysfunction [37]. In addition, Foxp3+ regulatory $\mathrm{T}$ cells (Tregs), which inhibit autoimmunity and protect against tissue injury, are decreased in the peripheral blood both in experimental models of obesity and individuals with type 2 diabetes [37, 40]. The generation of Tregs is reciprocally interconnected to that of proinflammatory IL-17-producing T helper (Th17) cells [41]. It is not known whether $\mathrm{T}$ helper cell subset polarisation occurs prior to the development of type 2 diabetes. Nevertheless, IL-6, a key cytokine in the generation of 
Th17 cells [41], is upregulated in adipose tissue in individuals with obesity [37, 42].

Our study has several strengths. The IRAS has a wellcharacterised sample population and uses validated measures of both insulin sensitivity and insulin secretion. In contrast to earlier reports, our regression models assessed the effects of major correlates of white cell count and established risk factors for type 2 diabetes (smoking, insulin sensitivity, insulin secretion, glucose tolerance, adiposity and chronic subclinical inflammation). The results were consistent across categories of sex, race/ethnicity, clinic, glucose tolerance and adiposity. The study also has limitations. The assay to measure insulin concentration has a significant interassay $\mathrm{CV}$. It is plausible that a more specific assay for insulin concentration could have resulted in a more precise assessment of the relationship between white cell count (or subfractions) and measures of insulin sensitivity and secretion. However, our method of measuring insulin sensitivity was validated against the euglycaemic-hyperinsulinaemic clamp [26]. A single determination of white cell count and differential is common practice in most epidemiological studies, but carries significant intraindividual variation: $11 \%$ for total white cell count, $7-16 \%$ for neutrophil count, $10-12 \%$ for lymphocyte count and $11-18 \%$ for monocyte count measurement [43]. Finally, white cell counts and subfractions were measured in each clinic centre. This might have contributed to ascertainment error of diabetic risk. Nevertheless, this type of error would tend to bias results towards the null hypothesis. Furthermore, the results were consistent across centres.

In summary, elevated total white cell, neutrophil and lymphocyte counts may be detected in individuals who are at increased risk of diabetes. The lymphocyte association was the strongest among the white cell types. This was partially explained by insulin sensitivity rather than low-grade inflammation, as measured by hsCRP. Future studies need to examine the dynamic interactions between white cell subfractions, macrophages and adipocytes in relation to weight gain and declining glucose tolerance.

Funding This study was supported by National Heart, Lung, and Blood Institute grants HL-47887, HL-47889, HL-47890, HL-47892 and HL-47902, and the General Clinical Research Centers Program (NCRR GCRC, M01 RR431 and M01 RR01346).

Duality of interest The authors declare that there is no duality of interest associated with this manuscript.

Contribution statement CL contributed to the conception and design of the study, analysis and interpretation of data and drafting the article, and gave final approval of the version to be published. AJH contributed to the analysis and interpretation of data and revised the manuscript critically for important intellectual content, and gave final approval of the version to be published. SMH contributed to the acquisition of data, analysis and interpretation of data and drafting the article, and gave final approval of the version to be published.

\section{References}

1. Donath MY, Shoelson SE (2011) Type 2 diabetes as an inflammatory disease. Nat Rev Immunol 11:98-107

2. Xu H, Barnes GT, Yang Q et al (2003) Chronic inflammation in fat plays a crucial role in the development of obesity-related insulin resistance. J Clin Invest 112:1821-1830

3. Wu H, Ghosh S, Perrard XD et al (2007) T cell accumulation and regulated on activation, normal $\mathrm{T}$ cell expressed and secreted upregulation in adipose tissue in obesity. Circulation 115:1029-1038

4. Larsen CM, Faulenbach M, Vaag A et al (2007) Interleukin-1receptor antagonist in type 2 diabetes mellitus. N Engl J Med 356: $1517-1526$

5. Goldfine AB, Fonseca V, Jablonski KA et al (2013) Salicylate (salsalate) in patients with type 2 diabetes: a randomized trial. Ann Intern Med 159:1-12

6. Solomon DH, Love TJ, Canning C, Schneeweiss S (2010) Risk of diabetes among patients with rheumatoid arthritis, psoriatic arthritis and psoriasis. Ann Rheum Dis 69:2114-2117

7. Dessein PH, Joffe BI, Stanwix A, Botha AS, Moomal Z (2002) The acute phase response does not fully predict the presence of insulin resistance and dyslipidemia in inflammatory arthritis. J Rheumatol 29:462-466

8. Gonzalez-Gay MA, Gonzalez-Juanatey C, Vazquez-Rodriguez TR, Miranda-Filloy JA, Llorca J (2010) Insulin resistance in rheumatoid arthritis: the impact of the anti-TNF-alpha therapy. Ann N Y Acad Sci 1193:153-159

9. Moreland LW, Baumgartner SW, Schiff MH et al (1997) Treatment of rheumatoid arthritis with a recombinant human tumor necrosis factor receptor (p75)-Fc fusion protein. N Engl J Med 337:141-147

10. Vozarova B, Weyer C, Lindsay RS, Pratley RE, Bogardus C, Tataranni PA (2002) High white blood cell count is associated with a worsening of insulin sensitivity and predicts the development of type 2 diabetes. Diabetes 51:455-461

11. Hanley AJ, Retnakaran R, Qi Y et al (2009) Association of hematological parameters with insulin resistance and beta-cell dysfunction in nondiabetic subjects. J Clin Endocrinol Metab 94:3824-3832

12. Festa A, D'Agostino R Jr, Howard G, Mykkänen L, Tracy RP, Haffner SM (2000) Chronic subclinical inflammation as part of the insulin resistance syndrome: the Insulin Resistance Atherosclerosis Study (IRAS). Circulation 102:42-47

13. De Rooij SR, Nijpels G, Nilsson PM et al (2009) Low-grade chronic inflammation in the relationship between insulin sensitivity and cardiovascular disease (RISC) population: associations with insulin resistance and cardiometabolic risk profile. Diabetes Care 32:1295-1301

14. Twig G, Afek A, Shamiss A et al (2013) White blood cells count and incidence of type 2 diabetes in young men. Diabetes Care 36:276-282

15. Schmidt MI, Duncan BB, Sharrett AR et al (1999) Markers of inflammation and prediction of diabetes mellitus in adults (Atherosclerosis Risk in Communities study): a cohort study. Lancet 353:1649-1652

16. Gkrania-Klotsas E, Ye Z, Cooper AJ et al (2010) Differential white blood cell count and type 2 diabetes: systematic review and metaanalysis of cross-sectional and prospective studies. PLoS One 5:e13405

17. Freeman DJ, Norrie J, Sattar N et al (2001) Pravastatin and the development of diabetes mellitus: evidence for a protective treatment effect in the West of Scotland Coronary Prevention Study. Circulation 103:357-362

18. Duncan BB, Schmidt MI, Pankow JS et al (2003) Low-grade systemic inflammation and the development of type 2 diabetes: the Atherosclerosis Risk in Communities Study. Diabetes 52:1799-1805

19. Chien K, Cai T, Hsu H et al (2009) A prediction model for type 2 diabetes risk among Chinese people. Diabetologia 52:443-450

20. Chao C, Song Y, Cook N et al (2010) The lack of utility of circulating biomarkers of inflammation and endothelial dysfunction for type 2 diabetes risk prediction among postmenopausal women: the 
Women's Health Initiative Observational Study. Arch Intern Med 170:1557-1565

21. Raynor LA, Pankow JS, Duncan BB et al (2013) Novel risk factors and the prediction of type 2 diabetes in the Atherosclerosis Risk in Communities (ARIC) study. Diabetes Care 36:70-76

22. Kaur H, Adams-Huet B, Smith G, Jialal I (2013) Increased neutrophil count in nascent metabolic syndrome. Metab Syndr Relat Disord 11: $128-131$

23. Meng W, Zhang C, Zhang Q et al (2012) Association between leukocyte and metabolic syndrome in urban Han Chinese: a longitudinal cohort study. PLoS One 7:e49875

24. Buyukkaya E, Karakas MF, Karakas E et al (2012) Correlation of neutrophil to lymphocyte ratio with the presence and severity of metabolic syndrome. Clin Appl Thromb Hemost. doi:10.1177/ 1076029612459675

25. Wagenknecht LE, Mayer EJ, Rewers M et al (1995) The Insulin Resistance Atherosclerosis Study: design, objectives and recruitment results. Ann Epidemiol 5:464-472

26. Saad MF, Anderson RL, Laws A et al (1994) A comparison between the minimal model and the glucose clamp in the assessment of insulin sensitivity across the spectrum of glucose tolerance. Insulin Resistance Atherosclerosis Study. Diabetes 43:1114-1121

27. Dot D, Miró J, Fuentes-Arderiu X (1992) Biological variation of the leukocyte differential count quantities. Scand J Clin Lab Invest 52: 607-611

28. Festa A, Williams K, Hanley AJ, Haffner SM (2008) Beta-cell dysfunction in subjects with impaired glucose tolerance and early type 2 diabetes: comparison of surrogate markers with first-phase insulin secretion from an intravenous glucose tolerance test. Diabetes 57:1638-1644

29. The Expert Committee on the Diagnosis and Classification of Diabetes Mellitus (2003) Follow-up report on the diagnosis of diabetes mellitus. Diabetes Care 26:3160-3167

30. Matthews DR, Hosker JP, Rudenski AS, Naylor BA, Treacher DF, Turner RC (1985) Homeostasis model assessment: insulin resistance and beta-cell function from fasting plasma glucose and insulin concentrations in man. Diabetologia 28:412-419

31. Harrell FE (2001) Regression modeling strategies. Springer, New York

32. Pratley RE, Wilson C, Bogardus C (1995) Relation of the white blood cell count to obesity and insulin resistance: effect of race and gender. Obes Res 3:563-571
33. Facchini F, Hollenbeck CB, Chen YN, Chen YD, Reaven GM (1992) Demonstration of a relationship between white blood cell count, insulin resistance, and several risk factors for coronary heart disease in women. J Intern Med 232:267-272

34. Targher G, Seidell JC, Tonoli M, Muggeo M, de Sandre G, Cigolini M (1996) The white blood cell count: its relationship to plasma insulin and other cardiovascular risk factors in healthy male individuals. J Intern Med 239:435-441

35. Sen N, Afsar B, Ozcan F et al (2013) The neutrophil to lymphocyte ratio was associated with impaired myocardial perfusion and long term adverse outcome in patients with ST-elevated myocardial infarction undergoing primary coronary intervention. Atherosclerosis 228:203-210

36. Alkhouri N, Morris-Stiff G, Campbell C et al (2012) Neutrophil to lymphocyte ratio: a new marker for predicting steatohepatitis and fibrosis in patients with nonalcoholic fatty liver disease. Liver Int 32: 297-302

37. Kanneganti TD, Dixit VD (2012) Immunological complications of obesity. Nat Immunol 13:707-712

38. Rotter V, Nagaev I, Smith U (2003) Interleukin-6 (IL-6) induces insulin resistance in 3T3-L1 adipocytes and is, like IL-8 and tumor necrosis factor-alpha, overexpressed in human fat cells from insulinresistant subjects. J Biol Chem 278:45777-45784

39. Kintscher U, Hartge M, Hess K et al (2008) T-lymphocyte infiltration in visceral adipose tissue: a primary event in adipose tissue inflammation and the development of obesity-mediated insulin resistance. Arterioscler Thromb Vasc Biol 28:1304-1310

40. Zeng C, Shi X, Zhang B et al (2012) The imbalance of Th17/ Th1/Tregs in patients with type 2 diabetes: relationship with metabolic factors and complications. J Mol Med (Berl) 90:175186

41. Bettelli E, Carrier Y, Gao W et al (2006) Reciprocal developmental pathways for the generation of pathogenic effector TH17 and regulatory T cells. Nature 441:235-238

42. Fried SK, Bunkin DA, Greenberg AS (1998) Omental and subcutaneous adipose tissues of obese subjects release interleukin-6: depot difference and regulation by glucocorticoid. J Clin Endocrinol Metab 83:847-850

43. Tang H, Jing J, Bo D, Xu D (2012) Biological variations of leukocyte numerical and morphologic parameters determined by UniCel DxH 800 hematology analyzer. Arch Pathol Lab Med 136:1392-1396 